\title{
Glutaric Acidemia Type 1
}

National Cancer Institute

\section{Source}

National Cancer Institute. Glutaric Acidemia Type 1. NCI Thesaurus. Code C99101.

A rare autosomal recessive inherited metabolic disorder caused by deficiency of the enzyme glutaryl-CoA dehydrogenase. It is characterized by abnormalities in the metabolism of lysine, hydroxylysine, and tryptophan that result in the accumulation and urinary excretion of glutaric acid. Patients present with brain atrophy, microcephaly, and acute dystonia. 